\title{
A Rare Case of Kimura Disease in A Black African
}

\section{Biram Codou Fall*, Assane Sall, El hadji Daouda Niang, Bamba Diaw, Ahmadou Gaye, Aly Salane, Aichetou Fall and Souhaibou Ndongo}

Department of Internal Medicine at Dalal Jamm Hospital, Dakar, Sénégal

*Corresponding Author: Biram Codou Fall, Department of Internal Medicine at Dalal Jamm Hospital, Dakar, Sénégal.
Received: December 13, 2021

Published: December 17, 2021

C) All rights are reserved by Biram Codou

Fall., et al.

\begin{abstract}
Introduction: Kimura disease or eosinophilic lymphogranuloma is a very rare condition. It occurs almost exclusively in patients of Far Eastern origin, but more rarely in Caucasians. It has not been described in black Africans. We report a case.

Observation: The patient was 66 years old, a farmer, not known to be a smoker, referred by an ENT specialist for investigation of a swelling of the left parotid region, evolving for 9 years, progressively increasing in volume, painless, accompanied by inflammatory episodes. The clinical examination revealed a patient in good general condition, apyretic, with normo colored conjunctiva. On inspection, there was a swelling of the left parotid region without inflammatory signs of the skin. On palpation, there was a swelling of firm consistency, poorly limited, painless, not flapping, mobile in relation to the superficial and deep planes, measuring approximately $7 \mathrm{~cm}$ in long axis. Palpation of the contralateral submaxillary region showed a smaller soft mass with a $3 \mathrm{~cm}$ long axis. Dermatological examination revealed prurigo. A cervicofacial CT scan showed an enlarged right parotid gland with irregular contours infiltrating the subcutaneous fatty tissue externally, without visualization of lithiasis or bone lysis. The biopsy of the accessory salivary glands revealed a grade 1 chronic sialadenitis. The biological workup was normal except for a major hypereosinophilia of 4400 . However, the systematic search for proteinuria was negative. The patient underwent a total right parotidectomy. Anatomopathological study of the specimen concluded to a Kimura disease. Oral corticosteroid therapy was instituted with a good evolution.

Conclusion: Kimura's disease is a rare condition, which usually affects Japanese men. It is characterized by subcutaneous nodules predominantly on the head and neck. The prognosis of the disease remains good.
\end{abstract}

Keywords: Kimura Disease; IgE; Lymphoid Tissue; Hyperplasia; Hypereosinophilia

\section{Introduction}

Kimura's disease or eosinophilic lymphogranuloma is a rare and benign chronic inflammatory disease of soft tissues and lymph nodes of unknown origin [1]. It was first described in China in 1937 but was not widely recognized until Kimura described it in the Japanese literature in 1948 [2]. The typical presentation is characterized by the triad: cervicofacial subcutaneous mass; blood hypereosinophilia with elevated serum total immunoglobulin E (IgE) levels; and the histopathologic features of follicular hyperplasia and reactive germinal centers with extensive eosinophilic infiltration. It is mainly reported in young men of Asian descent but may occur more rarely in Caucasian subjects [3]. Kimura disease is a rare condition, with approximately 300 cases reported to date [4] but very few cases have been described in Africa, particularly in North African subjects [5]. No cases have been reported in black Africans. We report a case of Kimura disease in a black Senegalese.

\section{Observation}

This was a 66-year-old patient, farmer and not known smoker, referred by an ENT specialist for exploration of a swelling of the left parotid region, evolving for 9 years, progressively increasing in volume, painless, accompanied by local inflammatory episodes wi- 
thout trismus or facial asymmetry (Figure 1). The patient reported perigandular, trunk and lower limb pruritus partially relieved by antihistamine. The clinical examination revealed a patient in good general condition, apyretic, with normo colored conjunctiva. On inspection, there was a swelling of the left parotid region without inflammatory signs of the skin. On palpation, there was a swelling of firm consistency, poorly limited, painless, not flapping, mobile in relation to the superficial and deep planes, measuring approximately $7 \mathrm{~cm}$ in long axis. Palpation of the contralateral submaxillary region showed a smaller soft mass with a long axis of $3 \mathrm{~cm}$ (Figure 2). Examination of the oral cavity showed no hyposialia or hemosialia or bulging of the posterolateral wall of the oropharynx. Prurigo was found on dermatological examination (Figure 3). The rest of the somatic examination was normal. The biological workup was normal except for a major hypereosinophilia at $4400 / \mathrm{mm}^{3}$. However, the systematic search for proteinuria was negative. Cervico-parotid ultrasound revealed an enlarged right parotid gland with heterogeneous echostructure. A cervicofacial CT scan showed an enlarged right parotid gland with irregular contours infiltrating the subcutaneous fatty tissue externally, without visualization of lithiasis or bone lysis. The biopsy of the accessory salivary glands was in favor of a grade 1 chronic sialadenitis. The patient had undergone a total right parotidectomy. Anatomopathological study of the lesions showed lymphoid tissue hyperplasia and massive eosinophilic infiltration compatible with Kimura disease. Oral corticosteroid therapy at $1 \mathrm{mg} / \mathrm{kg} / \mathrm{d}$ was initiated with progressive regression of the contralateral mass.

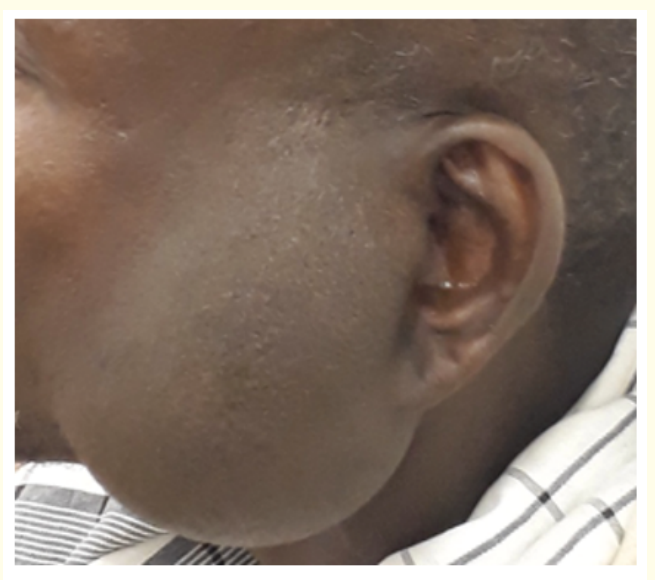

Figure 1: Voluminous Left Parotidomegaly.

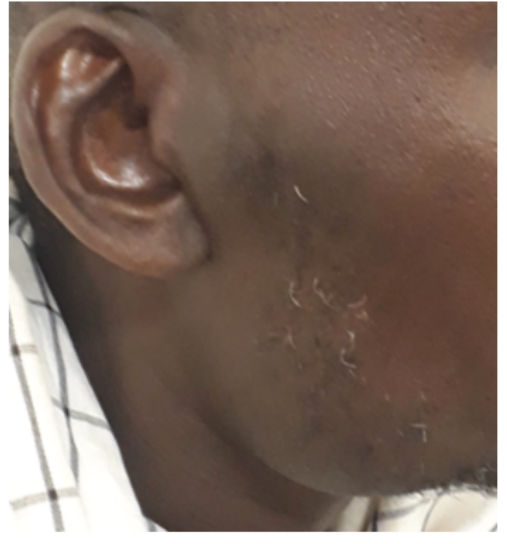

Figure 2: Sub-Maxillary Right Tumefaction.

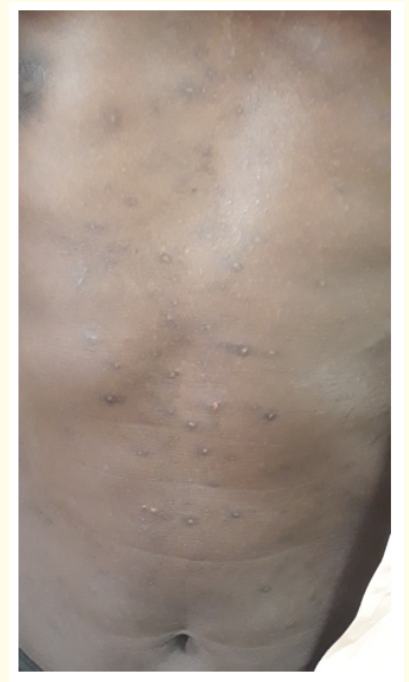

Figure 3: Prurigo.

\section{Discussion}

Kimura disease was first described in China by Kim in 1937 as eosinophilic hyperplastic lymphogranuloma [1]. Until 1948, the disease was widely recognized in Japan and consistently described by Kimura as «unusual granulations associated with hyperplastic changes in lymphoid tissue» [6]. It classically affects subjects from Southeast Asia where the disease is considered endemic, with a peak frequency in the third and fourth decades, a clear male predominance (sex ratio of $\mathrm{F} / \mathrm{H} 1 / 6$ ) [7] but the proportion of women 
affected increases with age (male to female ratio: overall, $4: 1 ;<20$ years, $17: 1 ; 20$ - 39 years, $4: 1$; and $\geq 40$ years, $2: 1 ; p=0.01$ ) [5]. Nevertheless, a 2004 study that included 21 patients with Kimura disease found a more heterogeneous ethnic distribution, with patients of European ancestry in seven cases, African American and Asian each in six cases, and Hispanic American and Arab each in one case [2]. Kakehi., et al. reported in 2018 about 278 observations published in the literature including 5 African cases [5] particularly in the Maghreb [8-10]. Our observation is atypical as it involves a 66-year-old black African patient of Senegalese origin. It is not clear from the literature whether genetic causes are associated with the etiopathogeny of Kimura disease. The late age of onset in our patient (66 years), which is higher than the average age in several series, can be explained in part by the latent evolution of the disease, the lack of knowledge of this condition in our tropical context and the multiplicity of differential diagnoses.

The etiopathogenesis of Kimura disease remains unknown at present. The endemicity in South-East Asia, the privileged involvement of the cervicocephalic sphere, the presence of a chronic inflammatory reaction associated with a hypereosinophilia and an increase in IgE antibodies plead in favor of a reactive process (immunologically or allergically mediated) to a particular stimulus not yet identified. Some authors have suggested a parasitic, allergic, autoimmune or viral origin [11]. The most suspected agents are the Epstein-Barr virus, which has been identified on several occasions by PCR in cutaneous and lymph node lesions [12], the HHV8 virus [13] and Candida Albicans [14]. According to the allergic theory, the stimulus induces IgE-induced type I hypersensitivity with secretion of eosinophil-activating cytokines. According to the autoimmune theory, the stimulus alters the immunoregulation of $\mathrm{T}$ lymphocytes releasing Th2-type cytokines (IL-4, IL-5, IL-13) [15].

The clinical presentation is often associated with subcutaneous nodules or plaques of variable size from 1 to $20 \mathrm{~cm}$ (average $3 \mathrm{~cm}$ ) [16]. The lesions are most often single (61. 8\%) or multiple (35. $3 \%$ [5]. Their consistency can be firm or soft. The lesions are not attached to the deep skin, but adhere to the overlying skin. Pruritus and melanin pigmentation of the overlying skin are frequently found $[17,18]$. Gao., et al. observed nerve infiltration by lymphocytes and eosinophils in patients with pruritus or pigmentation, explaining the possible pathogenesis of pruritus to some extent [19]. As in our patient, the preferential localization is cervico-facial $(76 \%)$, in particular subauricular and retroauricular with a recurrence of salivary gland involvement $[5,1]$. However, Chen $\mathrm{H}$., et al. have reported that non-Asian patients do not usually have salivary gland involvement [20]. Mucosal locations (gum, epiglottis, lip, palate, eye) [22] as well as visceral involvement (prostate, bladder, colon, liver) are exceptional [23,24], although forms with renal involvement are sometimes reported. Satellite lymph node involvement is often found with an incidence of 42 to $100 \%$ [25]. The disease can be complicated by asthma, allergic rhinitis, atopic dermatitis, vasculitis and arterial thrombosis, urticaria and other allergic symptoms $[5,15]$. The average delay in diagnosis is often several years [5] while that of our case is 9 years justified in large part by the rarity and the many differential diagnoses of this condition. Kimura disease can often be confused with lymphoma or Sjogren's syndrome, but its main differential diagnosis is eosinophilic angiolymphoid hyperplasia. Clinical and histologic differentiation between the 2 entities ALHE and Kimura disease is possible in most cases; typical features allow differentiation of the 2 clinical pictures [26]. However, there are still case reports and histopathologic publications that suggest a closer relationship between ALHE and Kimura disease [27].

Blood hypereosinophilia is almost constant (100\% of Zhang cases) [28] but variable in intensity, from $10 \%$ to $50 \%$, and hyper-IgE is very common [1]. Kimura disease is a subtype of essential hypereosinophilic syndrome (EHS), which is defined by eosinophilia with eosinophil-mediated organ damage and/or dysfunction, provided that other potential causes of damage have been excluded [29]. Some studies report that the number of eosinophils in the blood is closely proportional to the size of the lesion [30]. E. Kehkani., et al. found an increase in this hypereosinophilia with age and an average of 2000 elements $/ \mathrm{mm}^{3}$ was found in patients aged 40 years and over [5]. In a series of 10 cases of Asian patients, Iwahi., et al. suggested that major hypereosinophilia was a poor prognostic factor associated with more severe systemic involvement [31]. In our case, the major hypereosinophilia observed contrasts with the absence of visceral involvement. Recently, Ohta., et al. reported that serum eosinophilic cationic protein concentrations could be used as an additional parameter of disease activity [32]. In our patient, the systematic search for proteinuria is negative. Renal involvement is the main visceral manifestation observed in Kimura disease. Several associations between nephropathy and Kimura disease have been reported in the literature. Studies have shown that cytokine release in patients with Kimura disease can increase glomerular basement membrane permeability, causing proteinu- 
ria, which can ultimately lead to kidney damage. Others suggest persistent antigenemia leading to IgE deposits along the glomerular capillary membrane, with damage to the glomerulus explaining the proteinuria [33]. It should be remembered that IgE deposits have indeed been visualized by immunofluorescence along the capillaries and peri-mesangial areas [14].

Approximately 15 - 18\% of adult patients have renal involvement with proteinuria and 2/3 have a nephrotic syndrome [34]. Cases of necrotizing extracapillary glomerulonephritis associated with Kimura disease have also been reported [35].

Radiological investigations can be useful in Kimura disease to better define its extension. On ultrasonography, the mass may show heterogeneous or homogeneous echogenicity [36]. Som and Biller [37] explored by CT and MRI their patient with Kimura disease affecting the parotid gland and cervical lymph nodes. The CT scan showed contrast of the parotid gland and homolateral affected lymph nodes. The contrast of the affected areas being similar to that of the carotid artery, vascular involvement is thus suggested by the authors. In our patient, the radiological explorations were not specific and allowed a good appreciation of the extent of the lesions, however a vascular involvement was not demonstrated. Magnetic resonance imaging can differentiate its precise nature from other soft tissue tumors. This lesion tends to be heterogeneous, hypointense, and sometimes slightly hyperintense on T1 and $\mathrm{T} 2$ weighted imaging [38].

Pathologic examination of the lesions shows hyperplasia of lymphoid tissue with Florid germinal centers and massive eosinophilic infiltration. Germinal center necrosis, proteinaceous deposits, vascularization of germinal centers and sclerotic areas were also observed. Eosinophilic microabscesses were sometimes present [17]. The cutaneous histological presentation of Kimura disease remains quite monomorphic and confirmed the diagnosis in our patient. There are few studies that have focused on immunohistochemical examination of tissues in Kimura disease.

There are currently no uniform diagnostic criteria for Kimura disease. The following features should arouse suspicion: Young male, with a painless mass in the cervicofacial sphere with a chronic course; association of overlying skin pruritus and locoregional adenopathy; blood hypereosinophilia and elevated serum IgE level. On the basis of these clinical and biological manifestations, the diagnosis of Kimura disease can be considered in advance, but the final diagnosis is based on histological examination.

The treatment of Kimura disease is not codified. Surgery is proposed as the first line of treatment for single lesion forms, but recurrences are frequent. Corticosteroid therapy is by far the most frequently indicated medical treatment, especially in profuse forms or those inaccessible to surgery, or systematically in cases of renal involvement. In our patient, post-parotidectomy corticosteroid therapy showed a clear efficacy with a favorable evolution in agreement with several data in the literature. The efficacy of interferon, radiotherapy (low dose local irradiation, about 25 - $30 \mathrm{~Gy}$ ) especially post surgery has been reported in a few cases $[39,18]$. In one patient, a treatment associating prednisone and all-trans-retinoic acid allowed the remission of a cortico-dependent form [40]. Similarly the efficacy of cyclosporine [41] and Mofetil mycophenolate [42] have been reported about in a few rare cases. The prognosis of Kimura disease is generally good and no malignant transformation of the disease has been reported to date. However, the evolution can be marked by spontaneous remissions, but recurrences are frequent, noted in more than $25 \%$ of patients treated by surgery alone [43], usually between 1 and 3 years later [31].

\section{Conclusion}

Kimura disease is a rare and benign condition classically seen in subjects of Asian origin. However, the distribution is heterogeneous but it occurs exceptionally in black subjects. This rarity justifies in large part the diagnostic errors. This pathology should be evoked in front of any clinical presentation with cervico-facial mass associated with adenopathies and pruritus, hyper-eosinophilia with hyper-IgE. The evolution is generally benign, but the seriousness lies in the renal damage and the systematic search for proteinuria is essential.

\section{Bibliography}

1. Wang DY., et al. "Kimura Disease: A Case Report and Review of the Chinese Literature". Nephron Clinical Practice 111.1 (2008): c55-61.

2. Chen H., et al. "Kimura Disease: A Clinicopathologic Study of 21 Cases". The American Journal of Surgical Pathology 28.4 (2004): 505-513.

3. Googe PB., et al. "Kimura's disease and angiolymphoid hyperplasia with eosinophilia: two distinct histopathological entities". Journal of Cutaneous Pathology 14.5 (1987): 263-271. 
4. Li X., et al. "Misdiagnosed recurrent multiple Kimura's disease: A case report and review of the literature". Molecular and Clinical Oncology (2018).

5. Kakehi E., et al. "Kimura's disease: effects of age on clinical presentation". QJM: An International Journal of Medicine 113.5 (2020): 336-345.

6. Kung ITM., et al. "Kimura's disease: a clinico-pathological study of 21 cases and its distinction from angiolymphoid hyperplasia with eosinophilia". Pathology (Phila) 16.1 (1984): 39-44.

7. Chen H., et al. "Kimura Disease: A Clinicopathologic Study of 21 Cases". The American Journal of Surgical Pathology 28.4 (2004): 505-513.

8. Drouillard M., et al. "Kimura's disease in a 50-year-old Tunisian man". European Annals of Otorhinolaryngology, Head and Neck Diseases 134.2 (2007): 127-129.

9. Dezoteux F., et al. "Une localisation épiglottique asymptomatique inattendue dans le cadre d'une maladie de Kimura". Annales françaises d'oto-rhino-laryngologie et de pathologie cervico-faciale 136.1 (1996): 46-48.

10. Kettani M., et al. "Maladie de Kimura à localisation parotidienne: à propos d'un cas et revue de la literature". The Pan African Medical Journal 18.1 (2014).

11. Berney DM., et al. "Angiolymphoid hyperplasia with eosinophilia in the colon: a novel cause of rectal bleeding". Journal of Clinical Pathology 50.7 (1997): 611-613.

12. Nagore E., et al. "Detection of Epstein-Barr virus DNA in a patient with Kimura's disease". International Journal of Dermatology 39.8 (2000): 618-620.

13. Jang K-A., et al. "Polymerase chain reaction (PCR) for human herpesvirus 8 and heteroduplex PCR for clonality assessment in angiolymphoid hyperplasia with eosinophilia and Kimura's disease: HHV-8 and clonality in ALHE and Kimura's disease". Journal of Cutaneous Pathology 28.7 (2001): 363-367.

14. Takenaka T., et al. "Histological and immunological studies on eosinophilic granuloma of soft tissue, so-called Kimura's disease". Journal of Clinical Allergy 6.1 (1976): 27-39.

15. Kimura Y., et al. "Mast cells and T cells in Kimura's disease express increased levels of interleukin-4, interleukin-5, eotaxin and RANTES". Cytokines/chemokines in Kimura's disease. Clinical and Experimental Allergy 32.12 (2002): 1787-1793.
16. Sun Q-F., et al. "Kimura disease: review of the literature". Journal of Internal Medicine 38.8 (2008): 668-672.

17. Zhang $X$ and Jiao Y. "The clinicopathological characteristics of Kimura disease in Chinese patients". Clinical Rheumatology 38.12 (2019): 3661-3617.

18. Ye P., et al. "Comparison of the efficacy of different treatment modalities for Kimura's disease". Int Journal of Oral and Maxillofacial Surgery 46.3 (2017): 350-354.

19. Gao Y., et al. "Clinicopathologic study of parotid involvement in 21 cases of eosinophilic hyperplastic lymphogranuloma (Kimura's disease)". Oral Surgery, Oral Medicine, Oral Pathology, and Oral Radiology Endodontology 102.5 (2006): 651-658.

20. Chen H., et al. "Kimura Disease: A Clinicopathologic Study of 21 Cases". The American Journal of Surgical Pathology 28.4 (2004): 505-513.

21. Li T-J., et al. "Oral Surgery, Oral Medicine, Oral Pathology, and Oral Radiology Endodontology 82.5 (1996): 549-555.

22. Ray V., et al. "Maladie de Kimura à localisation palatine chez un Antillais". The Revue de Médecine Interne 24.4 (2003): 253-256.

23. Ranjith Kumar V., et al. "An Unusual Presentation of Recurrent Childhood Kimura Disease: Ileocecal Involvement". Pediatric Hematology and Oncology 43.5 (2021): e682-e684.

24. Berney DM., et al. "Angiolymphoid hyperplasia with eosinophilia in the colon: a novel cause of rectal bleeding". Journal of Clinical Pathology 50.7 (1997): 611-613.

25. Hui PK., et al. "Lymphadenopathy of Kimura's Disease". The American Journal of Surgical Pathology 13.3 (1989): 177-186.

26. Buder K., et al. "Angiolymphoide Hyperplasie mit Eosinophilie und Morbus Kimura - ein klinischer und histopathologischer Vergleich". Vergleich von ALHE und Morbus Kimura". JDDG: Journal der Deutschen Dermatologischen Gesellschaft 12.3 (2014): 224-228.

27. Helander SD., et al. "Kimura's disease and angiolymphoid hyperplasia with eosinophilia: New observations from immunohistochemical studies of lymphocyte markers, endothelial antigens, and granulocyte proteins". Journal of Cutaneous Pathology 22.4 (1995): 319-326.

28. Zhang., et al. "Thirty-five cases of Kimura's disease (eosinophilic lymphogranuloma): Correspondence". British Journal of Dermatology 139.3 (1998): 542-543. 
29. Valent P., et al. "Contemporary consensus proposal on criteria and classification of eosinophilic disorders and related syndromes". The Journal of Allergy and Clinical Immunology 130.3 (2012): 607-612.

30. Sakamoto M., et al. "Hematoserological analysis of Kimura's disease for optimal treatment". Otolaryngology-Head and Neck Surgery 132.1 (2005): 159-160.

31. Iwai H., et al. "Kimura Disease: Diagnosis and Prognostic Factors". Otolaryngology Neck Surgery 137.2 (2007): 306-311.

32. Ohta N., et al. "Serum concentrations of eosinophil cationic protein and eosinophils of patients with Kimura's disease". Allergology International 56.1 (2007): 45-49.

33. Akosa Ab., et al. "Kimura's Disease and Membranous Nephropathy". Nephron 58.4 (1991): 472-474.

34. Rajpoot DK., et al. "Nephrotic syndrome associated with Kimura disease”. Pediatric Nephrology 14.6 (2000): 486-488.

35. Chauvel C., et al. "Maladie de Kimura associée à une glomérulonéphrite extra-capillaire nécrosante". Annales de Dermatologie et de Vénéréologie 133.8-9 (2006): 689-692.

36. Ragu R., et al. "Kimura's Disease of the Parotid: A Complete Clinical-Radiological-Pathology Report". Medical Journal of Malaysia 69.4 (2004): 199-201.

37. Som P and Biller HF. "Kimura Disease Involving Parotid Gland and Cervical Nodes: CT and MR Findings". Journal of Computer Assisted Tomography 16.2 (1992): 320-322.

38. Oguz KK., et al. "Magnetic resonance imaging findings in Kimura's disease”. Neuroradiology 46.10 (2004): 855-888.

39. Kanny G., et al. "Une observation de maladie de kimura traitée par interféron et corticothérapie Générale". La Revue de Médecine Interne 20.6 (1999): 522-526.

40. Ben-chetrit E., et al. "Cetirizine: An effective agent in Kimura's disease". Arthritis and Rheumatology 53.1 (2005): 117-118.

41. Kaneko K., et al. "Successful treatment of Kimura's disease with cyclosporine". Journal of the American Academy of Dermatology 41.5 (1999): 893-894.

42. Shah K., et al. "Treatment of Kimura disease with mycophenolate mofetil monotherapy". JAAD Case Report 3.5 (2017): 416-419.
43. Baum EW., et al. "Angiolymphoid Hyperplasia with Eosinophilia. The Disease and a Comparison of Treatment Modalities". The Journal of Dermatologic Surgery and Oncology 8.11 (1982): 966-972.

\section{Assets from publication with us}

- Prompt Acknowledgement after receiving the article

- Thorough Double blinded peer review

- Rapid Publication

- Issue of Publication Certificate

- High visibility of your Published work

Website: www.actascientific.com/

Submit Article: www.actascientific.com/submission.php Email us: editor@actascientific.com

Contact us: +919182824667 